\title{
Fault-tolerant active phased array antenna with neural network
}

\author{
Svetlana Kondratieva ${ }^{1, *}$, Pavel Shmachilin ${ }^{1}$, and Natalia Anosova ${ }^{2}$ \\ ${ }^{1}$ Moscow Aviation Institute (National Research University), 125993 Moscow, Russia \\ ${ }^{2}$ The Peoples' Friendship University of Russia, 117198 Moscow, Russia
}

\begin{abstract}
A model and architecture for an active phased array antenna with a neural network control unit has been developed, which provides correction of the amplitude-phase distribution depending on the quality of work of individual array modules. The possibility of adapting the system to failures of individual modules is shown.
\end{abstract}

\section{Introduction}

The requirements for the global telecommunication systems being developed in recent years in the field of bandwidth and fault tolerance are constantly increasing. At the same time, there is an increasing need to provide such systems with antennas that are capable of adapting and automatically adjusting their parameters to changing working environment conditions. An example of such a system is the satellite system for global broadband Internet access. Mobile user stations should be equipped with an antenna array (AR) in the ground segment of such a system, providing automatic adjustment of the beam of the radiated pattern on the active (selected) communication satellite. At the same time, to ensure low cost, a rotary device can be provided in the system that can roughly direct the AR web to the selected satellite. Such a solution reduces the cost of mechanical operation of the rotator, and also reduces the requirements for the width of the scanning sector of the antenna array. The problem in this case is the need to automatically track the angular position of the satellite in the coordinate system of the AR when changing the direction of the carrier in space.

The implementation of such an AR is possible both with the partial use of digital signal processing methods in the last stages of the AR beam-forming network, and with the complete transition of all operations of the process of generating the radiation pattern into digital form with the signal digitization directly at the output of each antenna element.

Active development of Software-defined radio (SDR) systems in recent years makes it possible to implement a similar system using SDR devices, digital pattern formation and control algorithms in which PLD modules can be directly implemented.

\section{Neural network as a means of controlling a beam-forming network}

Active phased array antenna with digital antenna array is a large complex, dynamic system, the parameters of which change over time. As a result, this raises a challenge to tracking, monitoring and compensating for the degradation of characteristics.

*Corresponding author: kondratieff89@ya.ru 
The problem can be solved by classical (sharp analytical) or numerical methods. A subclass of numerical methods is "machine learning methods".

\subsection{AR Digital Part Model}

The structure of the receiving digital antenna array with quadrature analog-to-digital signal conversion can be represented in the form shown in fig. 1.

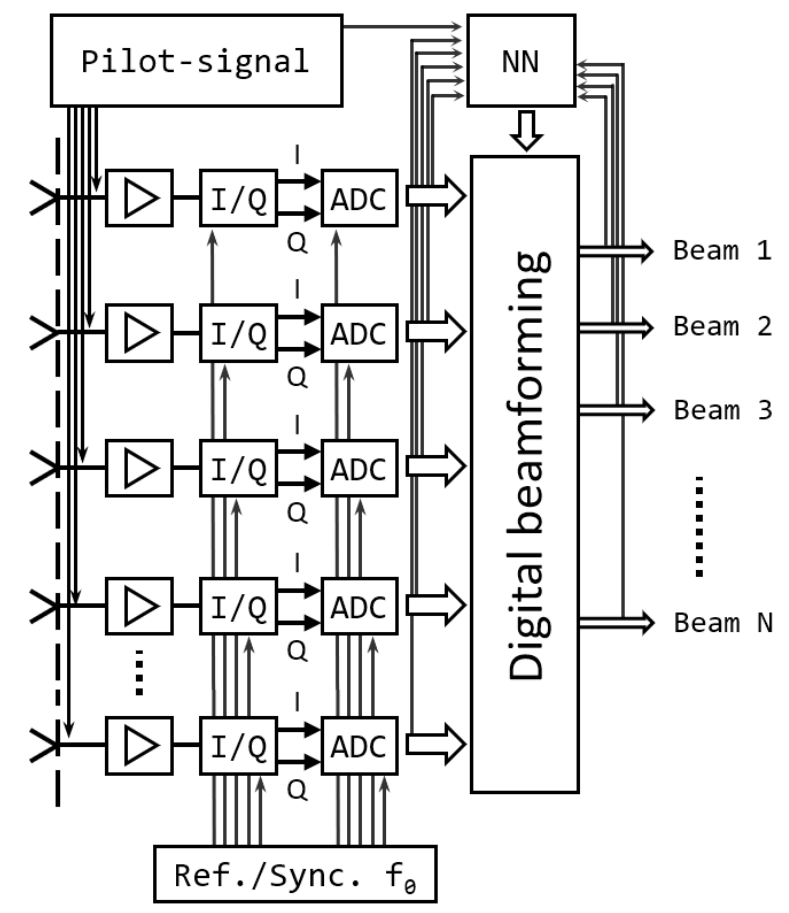

Fig. 1. Structure of digital array with neural network guidance module.

The signal from the antenna element in each channel passes through the analog part and through the quadrature analog-to-digital converter. Each stage of signal processing introduces its own distortion. The distortions of each AR channel are individual, and they change over time. The digital antenna array scheme should be able to compensate for channel non-similarity.

\subsection{The use of a pilot signal for detecting amplitude and phase discontinuity}

Existing active phased array antenna calibration algorithms imply the use of a special pilot signal, the characteristics of which can be estimated after it passes the AR channel. In this regard, a pilot signal of a special form, the characteristics of which are known in advance, can be brought to the AR inputs as close as possible to the antenna element. The analog part of the channel and the analog-to-digital converter will have the same effect on it as on the useful information signal. Thus, by detaching the received pilot signal from the mix of the input signal, it is possible to estimate the amplitude and phase distortion of each channel.

The problem, in this case, is aggravated by the fact that the useful signal and noise at the input of the antenna element will interfere with the calibration subsystem. This makes it difficult to apply rigorous analysis methods. But this problem can be solved using fuzzy logic and neural networks. 


\subsection{Beam-forming network}

The paper considers a model of the digital part of the array, where the inputs are the quadrature signals of the complex envelope obtained as a result of quadrature demodulation, the structure of which is shown in Fig. 2.

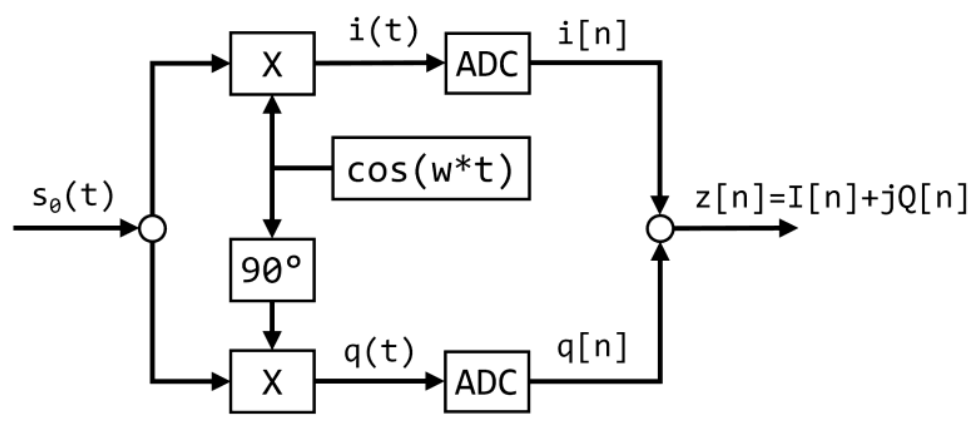

Fig. 2. Scheme of the quadrature demodulator of the complex envelope of the signal.

Thus, the signal at the ADC output is a vector of complex numbers. Vectors from all inputs can be combined into a signal matrix. The purpose of the beam-forming network is to coherently add signals from all the channels of the array for a given angular direction. The formation of a beam in a wide frequency band is hindered by the effect of the anglefrequency sensitivity of the beam.

$$
[z]=\left[\begin{array}{cccc}
z_{0}[0] & z_{1}[0] & \cdots & z_{K-1}[0] \\
z_{0}[1] & z_{1}[1] & \cdots & z_{K-1}[1] \\
\vdots & \vdots & \ddots & \vdots \\
z_{0}[N-1] & z_{1}[N-1] & \cdots & z_{K-1}[N-1]
\end{array}\right]
$$

To implement this algorithm, it's necessary to carry out beam formation at each frequency of the signal spectrum independently. For this, the decomposition of the signal matrix into the spectral one can be applied using the matrix Fourier transform. After that, the obtained matrix of spectra can be multiplied by a beam-forming matrix (2).

$$
\begin{gathered}
{\left[W_{F}\right](\theta, \varphi)=\left[\begin{array}{cccc}
w_{F}^{\theta, \varphi} & w_{F}^{\theta, \varphi} & \cdots & w_{F}^{\theta, \varphi}{ }_{0, K-1} \\
w_{F}^{\theta, \varphi}{ }_{1,0} & w_{F}^{\theta, \varphi}{ }_{2,2} & \cdots & w_{F}^{\theta, \varphi}{ }_{1, K-1} \\
\vdots & \vdots & \ddots & \vdots \\
w_{F}^{\theta, \varphi}{ }_{M-1,0}^{\theta, 0} & w_{F}^{\theta, \varphi}{ }_{M-1,2} & \cdots & w_{F}^{\theta, \varphi}{ }_{M-1, K-1}
\end{array}\right]} \\
w_{F}^{\theta, \varphi}{ }_{m, k}=e^{j \frac{2 \pi}{\lambda_{m}}\left[\left(\overrightarrow{\boldsymbol{x}}_{k} \mid \overline{\left.\left(\begin{array}{l}
\theta \\
)
\end{array}\right)\right)}\right]_{\times} \dot{A}_{m, k}\right.} \\
\left(\overrightarrow{\boldsymbol{X}}_{k} \mid \overrightarrow{\left(\begin{array}{l}
\boldsymbol{\theta} \\
\boldsymbol{\varphi}
\end{array}\right)}\right)=\left(\boldsymbol{x}_{k} \cos \varphi+\boldsymbol{y}_{k} \sin \varphi\right) \sin \theta+\mathbf{z}_{k} \cos \theta
\end{gathered}
$$

Each element of the beam-forming matrix (3) is defined as the projection of the placement vector of the antenna element in the array web on a given angular direction (4).

Multiplication of the beam-forming matrix by the matrix of spectra phases it, making the spectra in phase and allows their coherent addition. The resulting spectrum can be reconstructed by the inverse Fourier transform. 


\subsection{Neural network training}

By applying a mixture of the information signal and the pilot signal to the inputs of the antenna channels, the spectral matrix from the outputs of the ADC can be fed to the input of the neural network. Also, the output signal spectrum of the beam-forming network can be fed to the input of a neural network (see fig.3).

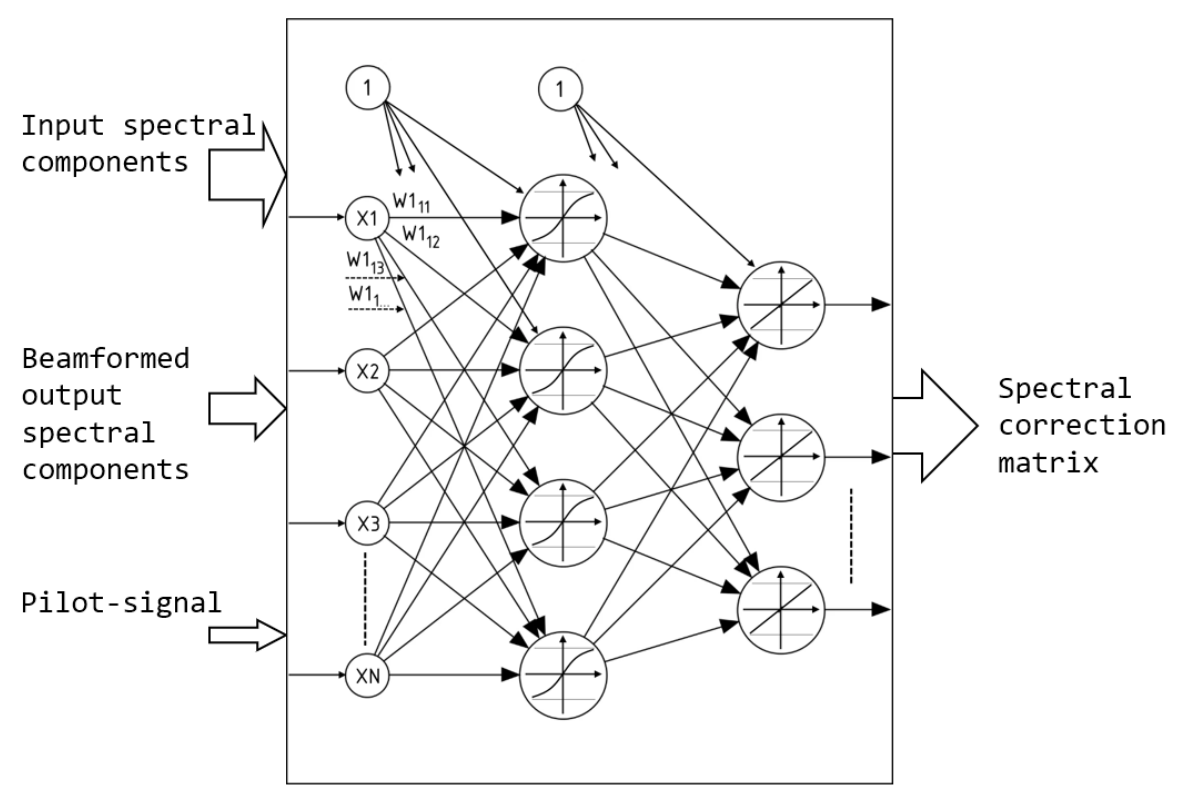

Fig. 3. Structure of neural network correction module.

A neural network can be trained on the basis of a "clean" pilot signal with predetermined possible amplitude and phase deviations of the antenna channels. In this case, the simplest topology of the direct distribution neural network and the error back propagation algorithm can be used. In this case, the amplitude and phase correction factors for each channel and each frequency will act as training samples which can be calculated analytically in the learning process.

\section{Conclusion}

Thus, applying the methods of spectral decomposition in each channel of the antenna array, you can use a neural network, the inputs of which are each spectral component of each antenna element, as well as the spectral output components of the beam-forming network. Having trained such a network to extract a matrix of correcting spectral coefficients for a beam-forming network on clean samples of a pilot signal, one can apply the obtained configuration of a neural network to generate correction coefficients for a mixture of a pilot signal, a useful information signal, and noise for the normal operating mode of the array. At the same time, the neural control module can be separated from the main digital network into a separate unit that operates at its own speed and doesn't require computing resources from the main digital network. The pilot signal can be mixed into the input signal of the array at rare times without disabling the main function of the system. It should also be noted that the training process of the neural network control module can be carried out on a separate prototype for a quite a long time in laboratory conditions; and the resulting network architecture is copied to existing system samples without loss of training 
time. An important feature of this approach is that the neural network in operation mode only works in direct feedforward mode. The time-consuming network learning process can and should be carried out at rare time intervals and on a separate sample.

\section{References}

1. Analysis of the effect of decorrelation of signals in the phased array antenna channels on the effectiveness of adaptive spatial filtering. Ratynskiy M.V. Radiotekhnika i elektronika. T. 62. No. 6. P. 546-553 (2017)

2. Galushkin A.I., Sudarikov V.A., Shabanov Ye.V. Neuromathematics, Methods for solving problems on neurocomputers. Matematicheskoye modelirovanie. No. 8. P. 93111 (1991)

3. Bashly P.N., Bogdanov V.M. The neural network method for solving the problem of optimizing the energy parameters of antenna arrays. Neyrokomp'yutery: razrabotka $\mathrm{i}$ primenenie. No. 11. P. 41-47 (2002) 